\title{
“PREKÄRES POTENTIAL" ISABELL LOREY: DIE REGIERUNG DER PREKÄREN
}

\author{
PHILIPP SPERNER, LAURIN LORENZ, MARLENE RADL, \\ LUDWIG FELHOFER, THOMAS OYSMÜLLER, \\ CHARLOTTE BOMERT
}

In ihrem schmalen Band "Die Regierung der Prekären” knüpft Isabell Lorey an gegenwärtige Debatten um den Begriff der Prekarität und den des Prekariats an. Dabei geht es ihr vor allem um die sozialphilosophische Dimension des Prekären, in dem sie einerseits die Grundlage der Regierung moderner Subjekte, zum anderen aber auch das Potential für politischen Widerstand erkennt.

Prekarität ist für Lorey keineswegs auf die gegenwärtig verbreitete Bedeutung im Sinne eines unsicheren Arbeitsverhältnisses beschränkt. Vielmehr versteht sie das Prekäre als grundlegende, nicht-hintergehbare Dimension des Lebens im Allgemeinen und somit als Basis für neue Formen der Gemeinschaftlichkeit. Sie beginnt dabei mit dem Entwurf eigener theoretischer Grundlagen, um im Anschluss daran eine Analyse der gegenwärtigen Funktionsweise des Kapitalismus zu liefern. Dabei bezieht sie sich auf Begrifflichkeiten verschiedenster Theorietraditionen. Lorey argumentiert, dass die Reproduktion gesellschaftlicher Machtstrukturen gegenwärtig durch eine Strategie stetiger Verunsicherung sichergestellt wird. Die Individuen sind der Regierung somit gerade in ihrer und durch ihre Prekarität unterworfen. Gleichzeitig jedoch, und das ist Loreys zentrale Wende, bietet die Prekarisierung auch die Möglichkeit zu einer neuen Form des Politischen. Diese würde das Subjekt gerade in seiner Prekarität zum Ausgangspunkt widerständigen Handelns und strategischer Zusammenschlüsse machen. Im letzten Teil des Buches führt Lorey diese Überlegungen zu einer Diskussion konkreter politischer

https://doi.org/10.14712/24646504.2021.9

(C) 2021 The Author. This is an open-access article distributed under the terms of the Creative Commons Attribution License (http://creativecommons.org/licenses/by/4.0). 
Praxis zusammen und erörtert, inwiefern verschiedene Formen der Prekarität die Basis für gemeinsame Formen des Widerständigen bieten können. Grundlage ihrer Argumentation stellt dabei die Unterscheidung von drei Dimension des Prekären dar, die sie insbesondere unter Bezugnahme auf Judith Butler und Michel Foucault entwickelt. Ausgangspunkt dieser Dreiteilung ist die Annahme einer existentiellen Abhängigkeit des Individuums. Dieses grundlegende Prekärsein ist das, was alle Individuen teilen, d.h. es ist das, was allen gemeinsam ist, aber gleichzeitig auch das, was sie von anderen separiert. Die Verletzlichkeit der Individuen, die sich in ihrem grundsätzlichen Prekärsein ausdrückt, ist nie gleich verteilt, sondern immer bereits in gesellschaftliche Hierarchien eingebunden. Diese hierarchisierte Differenzierung, die dem Prekärsein nicht nachgeordnet ist, sondern immer schon gleichzeitig mit diesem auftritt, bezeichnet Lorey als Prekarität. Im Unterschied zu den beiden vorangegangenen Dimensionen, die die sozialontologische Verfasstheit des Individuums beschreiben, analysiert Lorey mit dem Begriff der gouvernementalen Prekarisierung gegenwärtige Formen der Gemeinschaft und ihre historische Genese. Diese dritte Dimension des Prekären ist laut Lorey eng mit der Entwicklung einer spezifischen Weise des Regierens verbunden, die Individuen zunehmend selbst für die Absicherung ihres (Über-)Lebens verantwortlich macht und ihnen gleichzeitig die damit verbundenen Risiken überträgt. Wurden die negativen Folgen dieser zunehmenden Übertragung von Risiko und Verantwortlichkeit zunächst externalisiert bzw. auf die gesellschaftlichen Ränder verlagert, prägt die Prekarisierung laut Lorey inzwischen die Gesellschaft als Ganzes. Zugleich bedeute dies jedoch weder das Ende gesellschaftlicher Ungleichheit noch, dass alle Individuen gleichermaßen von den Folgen der Prekarisierung betroffen seien.

Neben diesem philosophischen Grundgerüst des Prekären führt Lorey eine Reihe weiterer Denker*innen ein, um die aktuelle Entwicklung der kapitalistischen Gesellschaft zu erfassen. Dabei knüpft sie häufig in eklektischer Weise an ideengeschichtlich aufgeladene Begriffe an, die sie unter anderen von Hobbes, Castel, Arendt, Marx, Deleuze, Spinoza und Virno übernimmt. Weder diskutiert sie diese Begriffe ausführlich, noch positioniert sie sich selbst eindeutig zu ihnen. Dennoch ist eine solche theoretische Diskussion auch kaum das Anliegen des Buches, vielmehr geht es ihr um das Aufzeigen neuer politischer Handlungsräume. Antworten auf die Frage nach neuen gemeinschaftlichen Praxen zieht sie aus den aktuellen Kämpfen um (Sorge-)Arbeit, wie aus jenen der spanischen Gruppe precarias a la deriva. Indem sie politische Handlungen betont, in denen spinozistische "Gemeinbegriffe" hergestellt werden sollen, wird politische Praxis der Theoriebildung vorgezogen. Dies wirkt verwunderlich, gerade weil sie am Anfang ihres Buches noch die von allen geteilte Gleichheit einer "nichthintergehbare[n] Gefährdetheit" 
(S. 26) betont und somit eine intelligible Vorstellung des menschlichen Seins über die materielle Erfahrung stellt. Eine Vermittlung dieser beiden erkenntnistheoretischen Positionen wäre jedoch dringend notwendig, um hieran anknüpfende Fragen beantworten zu können: Wie können "Gemeinbegriffe" konkret gebildet werden und wie können diese vermittelt werden? Werden sie diskursiv hergestellt und welchen Stellenwert hat dann Körperlichkeit?

Die utopische Reichweite ihres politischen Anliegens bleibt uneindeutig und scheint gegen Ende des Werkes stark eingeschränkt: Ist Widerständigkeit auf das Aufzeigen minimaler Praktiken und Veränderungen begrenzt oder geht es um das grundlegende Auflösen von Herrschafts- und Prekaritätsverhältnissen? Eher klingt Ersteres durch, was als sehr kleiner, erster Schritt in Richtung der Entwicklung neuer Sozialitäten gelesen werden kann. Die Möglichkeit zu solchen Schritten identifiziert sie in der virtuos-performativen Struktur der neuen Lebens- und Arbeitsverhältnisse, die zu einer Transformation der Beziehung von öffentlicher und privater Sphäre drängen. Öffentlichkeit wird aber nicht automatisch zu einem politischen Raum. Das Potential läge vielmehr darin, diese neue Form der Öffentlichkeit als politische zu gestalten.

Erkennt Lorey im Neoliberalismus die Tendenz, dass sich der Staat zunehmend über Unsicherheit stabilisiert, kann gefragt werden, ob sich diese Entwicklung in den letzten Jahren revidierte. Denn aktuell lässt sich ein Umschlag von der neoliberalen Erzählung rund um strikter Selbstverantwortung und postulierter Leistungsgerechtigkeit hin zu einem autoritären Staatsbild feststellen: Es wird vermehrt die Forderung nach einem Staat laut, der für die Sicherheit seiner Bürger*innen sowohl nach Innen wie nach Außen sorgen soll. Die zunehmende Prekarisierung von Lebens- und Arbeitsverhältnissen verschränkt sich nun mit einem zugespitzten Sicherheitsdiskurs im Inneren zu einer neuen Form autoritärer Staatlichkeit, die durch eine restriktive Asylpolitik die Prekarität bestimmter Personengruppen in extremer Geschwindigkeit vorantreibt. Es scheint, als hätte die "Stimme der Prekären" einen autoritären Ton angeschlagen. Diese Diagnose folgt aus Loreys Analyse keinesfalls zwingend, könnte aber den Zusammenschluss der prekarisierten Subjekte in unterschiedlichsten Lebenssituationen und die Forderung, neue politische Räume einzunehmen, unterstreichen.

LoRey, Isabell. Die Regierung der Prekären. Wien/Berlin: Turia + Kant 2012 [ISBN 978-3-85132-669-7] 